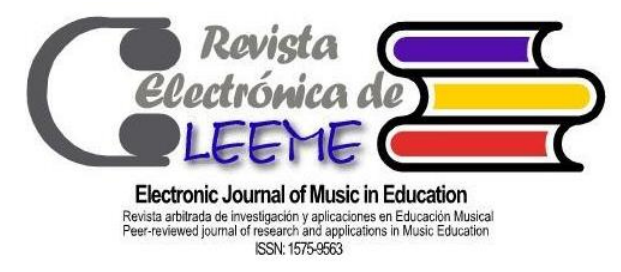

\title{
Análisis de materiales didácticos musicales para Primaria en la escuela digital
}

Analysis of musical didactic materials for Primary Education in digital school

Rosa María Vicente Álvarez rvicente@uvigo.es

Departamento de Didácticas Especiales

Universidad de Vigo

Orense, España

ORCID: https://orcid.org/0000-0001-7910-1362

\author{
Diana Marín Suelves \\ diana.marin@uv.es \\ Departamento Didáctica y Organización Escolar \\ Universidad de Valencia \\ Valencia, España \\ ORCID: https://orcid.org/0000-0002-5346-8665 \\ Olga Cepeda Romero \\ olceper@ull.edu.es \\ Departamento Didáctica e Investigación Educativa \\ Universidad de La Laguna \\ Tenerife, España \\ ORCID: https://orcid.org/0000-0002-5239-5038
}

doi: 10.7203/LEEME.42.10942

Recibido: 13-11-2017 Aceptado: 08-10-2018. Contacto y correspondencia: Rosa María Vicente Álvarez, Departamento de Didácticas Especiales Universidad de Vigo, Campus de Orense, 32004, Orense. España.

\section{Resumen}

Los materiales didácticos forman parte de la realidad educativa. El docente los selecciona en función del alumnado, el centro educativo, características o finalidad. En consecuencia, el material condiciona el método y homogeniza el proceso de enseñanza y aprendizaje. La realidad española de los últimos 30 años, a través de la especialización docente, ha hecho posible la evolución de los materiales escolares en educación musical. Este artículo proporciona respuestas a algunas preguntas sobre los cambios que suscita la utilización de recursos tecnológicos y digitales en educación y reflexiona sobre particularidades de uso en el aula de música. La investigación parte del análisis de contenido de una muestra de materiales para $5^{\circ}$ y $6^{\circ}$ de Educación Primaria. El conocimiento de los materiales didácticos digitales y musicales y sus estrategias pedagógicas ha sido posible gracias a la elaboración minuciosa de instrumentos de análisis específicos. Este estudio permite conocer las características tecnológicas, las tareas específicas y el tipo de aprendizaje musical que ofrecen editoriales y administración pública a través de la tecnología educativa.

Palabras clave: materiales digitales, educación musical, Educación Primaria.

\section{Abstract}

Didactic materials form part of educational practice. Teacher selects them according to their students, school, characteristics or purpose. Accordingly, the material determines the methodology and homogenizes the teaching and learning process. The Spanish reality of the last 30 years, through specialization in teaching, has made possible the evolution of school materials in music education. This article provides answers to some questions about the changes that the use of ICT resources brings about in education. The research starts from the analysis of content taken from samples of digital and musical didactic materials for 5th and 6 th grade of primary education. The knowledge of the digital and musical didactic materials and their pedagogical strategies has been possible thanks to the meticulous elaboration of specific analysis instruments. The study leads to knowledge of the technological characteristics, the specific tasks, and the type of musical learning that publisher and public administrations offer through educational technology.

Key words: didactic materials, digital, music education, Primary Education. 


\section{Introducción}

La tecnología educativa contribuye a la modificación de estrategias desarrolladas en el proceso de enseñanza y aprendizaje y en la forma de hacer del profesorado (Tejada y Pérez, 2016) y su introducción en las aulas de educación musical replantea la base pedagógica de la tarea docente invitando a emplear materiales y actividades musicales más comunicativas, significativas, funcionales y que respondan a la diversidad de alumnado presente en las aulas de hoy en día (Jubany, 2010). Sin embargo, los materiales digitales que replican las mismas estrategias que los materiales impresos no modifican la cultura profesional del profesorado ni los procesos desarrollados en las aulas. Es necesaria una evaluación exhaustiva y una difusión correcta de los materiales que permita visualizar las buenas prácticas (Serrano, 2017). Por otro lado, la transformación de los materiales didácticos digitales ha provocado importantes cambios en el ámbito educativo, reflejados en el diseño del currículum, el papel de las instituciones, la práctica docente o en las habilidades y procedimientos implicados en el proceso de aprendizaje (Peirats y Esnaola, 2015). La variedad y diversidad de materiales existentes hace necesario clarificar la tipología de recursos digitales para determinar su calidad. El tipo de recurso que configura la muestra de este estudio es la conceptualización del Material Didáctico Digital (MDD) como:

\footnotetext{
"Un paquete estructurado didácticamente de objetos digitales en línea dirigido a facilitar al alumnado el desarrollo de experiencias de aprendizaje en torno a una unidad de saber o competencia (...). Son materiales para la educación formal ya que en los mismos subyace una propuesta o proyecto de desarrollo curricular (...)" (Cepeda, Gallardo y Rodríguez, 2017, p. 83-84).
}

La hipótesis de partida es que "los materiales digitales permiten que los docentes puedan personalizarlos y adaptarlos a las características de su alumnado creando entornos de aprendizaje enriquecidos y variados. Los materiales digitales articulan nuevas formas de interacción de los aprendices con el conocimiento" (Area, 2016). Dicha premisa permite analizar la calidad de una muestra de recursos del área de Música para conocer las posibilidades de mejora educativa.

Este estudio parte del trabajo desarrollado desde tres universidades españolas de las Comunidades Autónomas de Galicia, Comunidad Valenciana e Islas Canarias. Cada una de ellas, dentro de sus competencias, elige el modelo de digitalización de la escuela que le resulta económicamente sostenible y centrado en la creación de un ecosistema digital de ámbito nacional que permita el normal desarrollo de sus opciones autonómicas (Area, Alonso, Correa, Del Moral, De Pablos, Paredes, et al., 2014).

\section{Contexto}

España se incorpora al cambio tecnológico en la década de los ochenta, cuando la administración pública se interesa por la aplicación de las TIC en la escuela. A finales de los 
noventa, se implementan programas completos que proporcionarán las infraestructuras necesarias de conexión para la mayor parte de los centros educativos. En 2010, se produce un nuevo giro en las aulas con el Programa 2.0 que impulsa el uso de las tecnologías educativas en las aulas de todo el país; aunque la Comunidad Valenciana no se incorpora a la implantación de dicho programa estatal, la comunidad canaria lo hace parcialmente y la gallega de manera completa. Las administraciones educativas, en sus políticas autonómicas, incorporan líneas de actuación que caminan en la dirección de la dotación de libros digitales a las escuelas y en la creación de espacios digitales propios (web escolar, aula virtual, etc.) para el aprendizaje escolar (Pereiro y Eirín, 2017).

Diversos estudios sobre tecnología educativa dan importancia a las repercusiones de la implantación y utilización de la tecnología educativa (Espigares, 2009; Tejada y Pérez, 2016). Evoluciones similares parecen haberse producido en las diferentes comunidades autónomas con similares resultados en los materiales. Faltan estudios que aborden el análisis de los materiales didácticos musicales y digitales. Basamos este estudio en la necesidad de conocer los materiales que se utilizan en las aulas, ya que propician un modelo docente determinado y condicionan los métodos musicales utilizados con el alumnado (Vicente, 2017).

Hablar de materiales didácticos y musicales es hablar de un compendio de recursos educativos que, por lo general van más allá de un curso académico o de un contenido en concreto. Oriol, Sustaeta y Ráez (1993) proponen una las primeras guías de recursos educativos, y Díaz (1998) expone una serie de materiales para la enseñanza musical que constituyen la referencia en las aulas escolares. El siglo XXI, ofrece estudios basados en el análisis descriptivo de los materiales musicales específicos (Romero, 2003; Vicente, 2009), análisis sobre la percepción docente (Rodríguez y Vicente, 2015) o programas de intervención que ponen de relieve la importancia del desarrollo musical (Tejada y Pérez, 2016) entre otros.

Analizamos materiales elegidos por el profesorado, considerando su forma y contenido, datos que proporcionan información sobre aspectos metodológicos, con el fin de reflexionar sobre lo común y comprender si dichos materiales promocionan buenas prácticas (Giráldez, 2010).

\section{Hipótesis}

El desarrollo discursivo de este estudio se desarrolla en tres ejes:

- La oferta digital de materiales didácticos musicales en diferentes formatos (web, repositorio, libro digital, recurso interactivo) representa las diversas posibilidades que ofrece la tecnología educativa, pero no necesariamente al servicio de la didáctica musical. 
- El uso de la tecnología educativa no representa un cambio significativo en la metodología, los materiales se presentan estáticos y con pocas posibilidades de modificación en un aula diversa y cambiante.

- Los materiales didácticos y digitales reproducen el método tradicional expositivo y en la educación musical condicionan las tareas grupales.

\section{Método}

Este estudio se basa en metodología cualitativa. Para la recolección de datos se ha realizado un estudio múltiple de casos a través de la compilación de casos individuales. La comparación entre los casos individuales requiere, entre otros aspectos, compartir características similares (Strauss y Corbin, 1998). Las características que comparten estos casos son: un marco teórico común, un instrumento y un procedimiento de análisis de la información. Éste último se desarrolla mediante el análisis de contenido como "conjunto de técnicas de análisis comunicativas usando procedimientos sistemáticos y objetivos para describir los contenidos de los mensajes" (Bardin, 2004, p. 29). En cuanto a los materiales seleccionados responden a la diversidad de recursos encontrados en las aulas de música. Y, la muestra está compuesta por materiales musicales de Primaria utilizados en tres comunidades autónomas españolas.

El objetivo principal del trabajo presentado es conocer las estrategias metodológicas que ofrecen los materiales didácticos digitales y musicales, a través de la descripción y comparación de las características de los materiales que reflejan la diversidad de la oferta actual para la educación musical.

\subsection{Muestra}

Este estudio analiza materiales para los cursos de $5^{\circ}$ y $6^{\circ}$ de Educación Primaria del área de Educación Musical, presentes en plataformas institucionales de las comunidades autónomas y plataformas comerciales y redes educativas y/o asociaciones profesionales. Los criterios acordados para la selección de esta muestra analizada son:

1) El análisis de materiales se fija en los cursos $5^{\circ}$ y $6^{\circ}$ de Primaria.

2) Los MDD deben suponer propuestas estructuradas de aprendizaje para el alumnado encontrados en diferentes entornos de aprendizaje digital.

3) Materiales de diferentes materias: para nuestro fin, seleccionamos los de la materia de educación musical.

4) Las dimensiones contempladas en el instrumento de análisis deben ser analizables a través del instrumento.

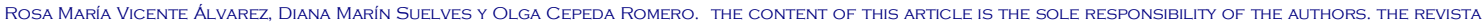
ELECTRÓNICA DE LEEME AND UNIVERSITAT DE VALËNCIA ARE NOT LIABLE FOR ANY LEGAL ACTIONS THAT MAY ARISE INVOLVING THE ARTICLE'S CONTENT, REVISTA ELECTRÓNICA DE LEEME - LISTA ELECTRONICA EUROPEA DE MUUSICA EN LA EDUCACION- HTTP://OJS.UVES/INDEX.PHP/LFEME/INDEX ISSN: 1575-9563 EDITORES: UNIVERSIDAD DE VALENCIA Y JESÚS TEJADA GIMÉNEZ. VISIBILIDAD DE ESTA REVISTA: SCOPUS, EMERGING SOURCES CITATION INDEX (CLARIVATE), EBSCO, CINDOC (CSIC), CITEFACTOR, COPAC, DIALNET, DICE (CSIC), DOAJ, E-REVISTAS (CSIC), EBSCO PREMIER, ERIH+, GALE CENGAGE LEARNING, IN-RECS, IRESIE, LATINDEX, MIAR, OCLC WORLDCAT, RESH, REDIB, RILM CORE JOURNALS, SUDOC, ULRICHS, ESTA REVISTA ESTÁ PUBLICADA CON EL APOYO INSTITUCIONAL DE REDIRIS-CONSEJO SUPERIOR DE INVESTIGACIONES CIENTÍFICAS Y ES DE ACCESO LIBRE. CREATIVE COMMONS LICENSE $4.0 \mathrm{BY}$ 
La Tabla 1 muestra la distribución de las plataformas institucionales, comerciales y redes profesionales seleccionadas para aportar materiales de análisis. A continuación, presentamos los ejemplos de los materiales analizados:

Tabla 1. Distribución de la muestra

\begin{tabular}{|c|c|c|c|c|c|}
\hline & & \multicolumn{3}{|c|}{ Tipo de portal o plataforma } & \multirow[b]{2}{*}{ Total } \\
\hline & & Institucionales & Comerciales & $\begin{array}{c}\text { Redes } \\
\text { educativas }\end{array}$ & \\
\hline \multirow[t]{3}{*}{ Muestra } & $\mathrm{N}^{\circ}$ de portales o plataformas & 6 & 14 & 7 & 27 \\
\hline & $\mathrm{N}^{\mathrm{o}}$ de recursos analizados & 17 & 32 & 19 & 68 \\
\hline & Total & 23 & 46 & 26 & \\
\hline
\end{tabular}

Fuente: Cepeda, Gallardo y Rodríguez (2017)

El procedimiento desarrollado para el análisis de las plataformas y MDD de la muestra se llevó a cabo a través de la organización de grupos de trabajo con las siguientes fases:

- Constitución de grupos de trabajo.

- Asignación de plataformas a analizar.

- Selección de los MDD a evaluar.

- Elaboración de un informe individual.

- Contrastación y elaboración del informe final de cada MDD.

- Discusión y elaboración del Informe Final de cada Comunidad Autónoma.

- Disponibilidad de los documentos finales en el espacio virtual del Proyecto.

El primer material es la plataforma digital de una editorial de libros de texto. Las editoriales y el libro de texto tienen un peso enorme en la escuela. El análisis de una plataforma digital de libro de texto ofrece la imagen del uso generalizado de las TIC en el aula de Música en Educación Primaria, a través de la actualización de un material tradicional. Hemos analizado el material interactivo y el libro digital, que se complementa con el impreso.

El segundo material analizado pertenece a la plataforma institucional de la comunidad autónoma de Valencia (Mestre a casa). La mayor parte de las políticas educativas apuestan por la creación de espacios web como repositorio institucional, aspecto por otro lado ya generalizado en los centros educativos, a través de sus webs, por ello, analizamos un recurso encontrado en este tipo de entornos.

El tercer material analizado ofrece un ejemplo de recursos de acceso abierto ubicado en un repositorio conocido y utilizado de forma generalizada en el contexto español. Las redes educativas de acceso abierto presentan una secuencia relacionada con el currículo donde la mayor parte de los materiales ofrecidos en la plataforma no siempre están contextualizados en un nivel curricular de la educación musical. Este material ejemplifica el uso paralelo de fuentes complementarias.

Rosa MARIA VICENTE ÁlVAREZ, DIANA MARIN SUELVES Y Olga CEPEDA ROMERO. THE CONTENT OF THIS ARTICLE IS THE SOLE RESPONSIBILITY OF THE AUTHORS. THE REVISTA ELECTRÓNICA DE LEEME AND UNIVERSITAT DE VALĖNCIA ARE NOT LIABLE FOR ANY LEGAL ACTIONS THAT MAY ARISE INVOLVING THE ARTICLE'S CONTENT REVISTA ELECTRÓNICA DE LEEME - LISTA ELECTRÓNICA EUROPEA DE MUSICA EN LA EDUCACION- HTTP://OJS UVES/INDEXPHP/LFEME/INDEX ISSN: 1575-9563 EDITORES: UNIVERSIDAD DE VALENCIA Y JESÚS TEJADA GIMÉNEZ. VISIBILIDAD DE ESTA REVISTA: SCOPUS, EMERGING SOURCES CITATION INDEX (CLARIVATE), EBSCO, CINDOC (CSIC), CITEFACTOR, COPAC, DIALNET, DICE (CSIC), DOAJ, E-REVISTAS (CSIC), EBSCO PREMIER, ERIH+, GALE CENGAGE LEARNING, IN-RECS, IRESIE, LATINDEX, MIAR, OCLC WORLDCAT, RESH, REDIB, RILM CORE JOURNALS, SUDOC, ULRICHS, ESTA REVISTA ESTÁ PUBLICADA CON EL APOYO INSTITUCIONAL DE REDIRIS-CONSEJO SUPERIOR DE INVESTIGACIONES CIENTÍFICAS Y ES DE ACCESO LIBRE. CREATIVE COMMONS LICENSE 4.0 BY 


\subsection{Instrumento}

La elaboración del instrumento de análisis supuso un proceso de reflexión y discusión progresivo por parte del equipo de investigación del Proyecto Escuel@ Digit@1 (Cepeda, Gallardo y Rodríguez, 2017). Presentamos las dimensiones de las variables del instrumento y una breve descripción de cada una:

(1) Datos de identificación del evaluador.

(2) Características identificadoras del material o recursos educativos: clasificación y agrupamiento.

(3) Estructura del material: información sobre el formato externo, imagen ofrecida y posibilidades visuales y sonoras.

(4) Dimensión tecnológica: descripción de la base informática y de las necesidades de la infraestructura tecnológica para su uso en el aula.

(5) Dimensión de diseño: necesidad de conocer navegabilidad, facilidades o dificultades de movimientos e intuición en el desarrollo de la actividad.

(6) Dimensión pedagógica: cuestión didáctica que subyace en el uso de los recursos y posibilidades escolares. Analizar los recursos metodológicos empleados.

(7) Dimensión de contenido: centrada en los aspectos curriculares.

(8) Evaluación y seguimiento: aspectos de mejora y su uso con fines pedagógicos.

(9) Comentarios finales: fortalezas y debilidades del MDD analizado.

Una vez determinado el instrumento, para su validación definitiva, se llevó a cabo la aplicación piloto, por grupos de tres personas para posibilitar la triangulación de datos y una revisión final del instrumento y sus variables por un total de 8 expertos externos (5 internacionales y 3 de universidades nacionales).

\subsection{Procedimiento}

El protocolo seguido para el desarrollo del procedimiento ha sido exhaustivo y ha seguido aspectos vertidos en Santana, Eirín y Marín (2017), que presentan una revisión de distintos estudios desarrollados en el ámbito educativo y que han elaborado sistemas de evaluación de portales y recursos digitales más o menos complejos (Cepeda, Gallardo y Rodríguez, 2017; Fernández-Pampillón, Domínguez, y Armas, 2011).

El trabajo de campo parte del análisis de contenido de diferentes materiales utilizados en las tres comunidades autónomas españolas citadas. Los datos son extraídos por las investigadoras que desarrollan su análisis de manera independiente. La información se vierte en matrices de igual formato con idénticos criterios de recogida de datos, con el fin de obtener una visión global de la realidad escolar y de los materiales utilizados para la enseñanza musical. El

ROSA MARIA VICENTE ÁlVAREZ, DIANA MARIN SUELVES Y Olga CEPEDA ROMERO. THE CONTENT OF THIS ARTICLE IS THE SOLE RESPONSIBILITY OF THE AUTHORS. THE REVISTA ELECTRÓNICA DE LEEME AND UNIVERSITAT DE VALĖNCIA ARE NOT LIABLE FOR ANY LEGAL ACTIONS THAT MAY ARISE INVOLVING THE ARTICLE'S CONTENT REVISTA ELECTRÓNICA DE LEEME - LISTA ELECTRONNCA EUROPEADE MUSICA EN LA EDUCACION- HTTP://OJSUVES/INDEX.PHP/LEEME/INDEX ISSN: 1575-9563 EDITORES: UNIVERSIDAD DE VALENCIA Y JESÚS TEJADA GIMÉNEZ. VISIBILIDAD DE ESTA REVISTA: SCOPUS, EMERGING SOURCES CITATION INDEX (CLARIVATE), EBSCO, CINDOC (CSIC), CITEFACTOR, COPAC, DIALNET, DICE (CSIC), DOAJ, E-REVISTAS (CSIC), EBSCO PREMIER, ERIH+, GALE CENGAGE LEARNING, IN-RECS, IRESIE, LATINDEX, MIAR, OCLC WORLDCAT, RESH, REDIB, RILM CORE JOURNALS, SUDOC, ULRICHS, ESTA REVISTA ESTÁ PUBLICADA CON EL APOYO INSTITUCIONAL DE REDIRIS-CONSEJO SUPERIOR DE INVESTIGACIONES CIENTIFICAS Y ES DE ACCESO LIBRE. CREATIVE COMMONS LICENSE $4.0 \mathrm{BY}$ 
análisis parte de una descripción de las características del material a nivel tecnológico y de las tareas que ofrece según las posibilidades didácticas. Los puntos tratados son:

- El análisis de los materiales didácticos seleccionados siguiendo las sugerencias relacionadas con el análisis de contenido.

- La elaboración de los informes correspondientes siguiendo la misma estructura que la establecida en el protocolo de análisis de los MDD y puesta en común de los equipos de investigación siguiendo el procedimiento comentado anteriormente.

\section{Resultados}

Las Tablas 2, 3, 4 y 5 describen y analizan los materiales, en función de las dimensiones y variables formuladas en el instrumento de análisis:

Tabla 2. Material 1. A. Análisis de material editorial (libro de texto digital)

\begin{tabular}{ll}
\hline Dimensión & \multicolumn{1}{c}{ Principales características } \\
\hline Identificación & Libro de texto digital (Galicia, 2014). \\
\hline Estructura & Entorno de acceso restringido exclusivo docente. Estructura similar al libro impreso. \\
\hline Tecnología & $\begin{array}{l}\text { Acceso y manipulación del entorno cerrado a través de la web, requiere contraseña, facilitada al } \\
\text { centro que adquiere esos materiales, por petición docente. }\end{array}$ \\
\hline Diseño & $\begin{array}{l}\text { Navegación fácil e intuitiva apoyada en iconos, lineal y paginada. Existe un menú contextual. } \\
\text { La velocidad de carga es buena, rápida y sin problemas. } \\
\text { Materiales complementarios: CD-ROM, CD musical, libro de fichas impreso a color, libro de } \\
\text { fichas para la evaluación, materiales en cartón, mascota, etc. } \\
\text { Estructura: presentación, cuerpo de contenidos en secciones y actividades o ejercicios. }\end{array}$ \\
\hline Pedagogía & $\begin{array}{l}\text { Recursos claros y formato de libro de texto tradicional. } \\
\text { Concepción expositiva, secuencial y transmisora del conocimiento. La calidad del formato es } \\
\text { adecuada, clara y sencilla, dosificada textualmente. } \\
\text { Contiene recursos fuera de la plataforma online, es necesario contar con el material impreso. }\end{array}$ \\
\hline Contenido & $\begin{array}{l}\text { Expositor de materiales sin apartado docente o de gestión. } \\
\text { No contempla evaluación progresiva. Desarrolla una autoevaluación continua. }\end{array}$ \\
\hline Evaluación y & $\begin{array}{l}\text { Contempla acciones propias de un libro de texto tradicional. } \\
\text { No existe la opción de crear recursos personalizables o de gestión de información. El alumno es } \\
\text { un receptor y/o repetidor de tareas hasta realizarlas de modo correcto, obteniendo recompensas. }\end{array}$ \\
\hline Fuento Elations
\end{tabular}

Fuente: Elaboración propia

Tabla 3. Material 1. B y C. Análisis de material editorial (libro de texto digital)

\begin{tabular}{lc}
\hline \multicolumn{1}{c}{ Dimensión } & Principales características \\
\hline Identificación & Material interactivo musical (Galicia, 2014). \\
\hline
\end{tabular}

Rosa MARIA VICENTE ÁlVAREZ, DIANA MARÍN SUELVES Y OLGa CEPEDA ROMERO. THE CONTENT OF THIS ARTICLE IS THE SOLE RESPONSIBILITY OF THE AUTHORS. THE REVISTA ELECTRÓNCA DE LEEMEAND UNVERSTAT DE VALENCIA YRE NOT CEABL FOR ANYLEGAL ACTIONS THAT MAY ARISE INVOLVING THEERTICLE'S CONTENT REVISTA ELECTRÓNICA DE LEEME - UISTA ELECTRONICA EUROPEA DE MUUSICA EN LA EDUCACION- HTPP//ONYLEGACSINDEX PHP/ UAYEME/INDEXISSN: 1575-9563 EDITORES: UNIVERSIDAD DE VALENCIAY JESÚS TEJADA GIMÉNEZ. VISIBILIDAD DE ESTA REVISTA: SCOPUS, EMERGING SOURCES CITATION INDEX (CLARIVATE), EBSCO, CINDOC (CSIC), CITEFACTOR, COPAC, DIALNET, DICE (CSIC), DOAJ, E-REVISTAS (CSIC), EBSCO PREMIER, ERIH+, GALE CENGAGE LEARNING, IN-RECS, IRESIE, LATINDEX, MIAR, OCLC WORLDCAT, RESH, REDIB, RILM CORE JOURNALS, SUDOC, ULRICHS, ESTA REVISTA ESTÁ PUBLICADA CON EL APOYO INSTITUCIONAL DE REDIRIS-CONSEJO SUPERIOR DE INVESTIGACIONES CIENTIFICAS Y ES DE ACCESO LIBRE. CREATIVE COMMONS LICENSE 4.O BY 


Estructura
Presenta dos materiales diferentes.
digital. Tiene enlaces externos.
Material C. Unidad didáctica. Contenidos integrados en tareas interactivas y en
formato digital.

\begin{tabular}{ll}
\hline Tecnología & $\begin{array}{l}\text { Materiales iconográficos e hipertextuales (HTML y/o software Flash). Actividades } \\
\text { interactivas tienen sonido y movimiento. Destaca la imagen fija. } \\
\text { Navegación fácil e intuitiva. Velocidad adecuada y fluida. }\end{array}$ \\
\hline Diseño & $\begin{array}{l}\text { Material 2: Desarrollo de tareas sin necesidad de utilizar el recurso tecnológico. } \\
\text { Material 3: Compendio de tareas para trabajar de manera interactiva e individual. Los } \\
\text { ejercicios no requieren de tareas o recursos ajenos al material digital. }\end{array}$ \\
& Formato adecuado, claro y sencillo y dosificado textualmente. \\
\hline Pedagogía & $\begin{array}{l}\text { Pedagogía expositiva y transmisora de conocimiento, presenta los recursos de manera } \\
\text { clara y adopta el formato de libros de texto tradicional. }\end{array}$ \\
\hline Actividades organizadas secuencialmente, según dificultad. \\
\hline Contenido & $\begin{array}{l}\text { Material B. Contenidos musicales relacionados con el lenguaje musical, la } \\
\text { organología y aspectos del currículo de primaria. Secuencia en PDF. }\end{array}$ \\
& $\begin{array}{l}\text { Material C. Unidades temáticas con contenidos específicos musicales, compás, } \\
\text { matices, agrupamientos musicales, audición, etc. Secuencia en Flash. }\end{array}$ \\
\hline $\begin{array}{l}\text { Evaluación y } \\
\text { seguimiento }\end{array}$ & $\begin{array}{l}\text { Ambos materiales disponen de autoevaluación individual instantánea. } \\
\text { No disponen de herramientas docentes para el seguimiento del alumnado o gestión del } \\
\text { aprendizaje. }\end{array}$
\end{tabular}

Fuente: Elaboración propia

Los materiales analizados presentan una interfaz de navegación simple, aunque carecen de una organización o estructura planificada o diseñada con algún criterio pedagógico, pero al no disponer de muchos elementos (sólo cuenta con el libro impreso en formato PDF), su uso es sencillo.

Los materiales didácticos existentes son adecuados desde un punto de vista de una pedagogía expositiva. Tienen calidad técnica, de contenido, de ejecución y de aplicación del mismo en tareas concretas. Incorporan como elementos multimedia el sonido y la realización de algunas tareas de carácter conductista (ejercicios de unir con flechas o relacionar). Los ejercicios o actividades interactivas son similares en todas las unidades o lecciones, dependiendo del material analizado. La interactividad es limitada, se presentan algunos ejercicios online de manera individual o expositiva; además, los elementos multimedia son pocos y cumplen un papel poco significativo, o con finalidad de atracción. Su planteamiento metodológico es poco innovador, las actividades son de pregunta-respuesta, acierto-error o selección de respuesta correcta. La navegabilidad es buena, pero está limitada los recursos que son pobres desde el punto de vista digital.

Los materiales digitales analizados son libros de texto en formato PDF, presentados como materiales estructurados organizados secuencialmente en unidades o temas. Subyace una

Rosa MARIA VICENTE ÁlVAREZ, DIANA MARIN SUELVES Y Olga CEPEDA ROMERO. THE CONTENT OF THIS ARTICLE IS THE SOLE RESPONSIBILITY OF THE AUTHORS. THE REVISTA ELECTRÓNICA DE LEEME AND UNIVERSTAT DE VALËNCIA ARE NOT LABLE FOR ANY LEGAL ACTIONS THAT MAY ARISE INVOLVING THE ARTICLE'S CONTENT REVISTA ELECTRÓNUISTA LEEME - USTA ELECTRONICA EUROPEADE MUSICA EN LA EDUCACION- HTTP://OJSUVES/INDEXPHP//EFME/INDEXISSN: 1575-9563 EDITORES: UNIVERSIDAD DE VALENCIAY JESÚS TEJADA GIMÉNEZ. VISIBILIDAD DE ESTA REVISTA: SCOPUS, EMERGING SOURCES CITATION INDEX (CLARIVATE), EBSCO, CINDOC (CSIC), CITEFACTOR, COPAC, DIALNET, DICE (CSIC), DOAJ, E-REVISTAS (CSIC), EBSCO PREMIER, ERIH+, GALE CENGAGE LEARNING, IN-RECS, IRESIE, LATINDEX, MIAR, OCLC WORLDCAT, RESH, REDIB, RILM CORE JOURNALS, SUDOC, ULRICHS, ESTA REVISTA ESTÁ PUBLICADA CON EL APOYO INSTITUCIONAL DE REDIRIS-CONSEJO SUPERIOR DE INVESTIGACIONES CIENTIFICAS Y ES DE ACCESO LIBRE. CREATIVE COMMONS LICENSE 4.0 BY 
concepción de aprendizaje por recepción en gran grupo y de naturaleza individual. No hay nueva producción o generación de contenidos ya que lo ofertado en la plataforma es una reproducción digitalizada de materiales en formato tradicional (libros de texto o enciclopedias), con la presentación de ejercicios o actividades que son habituales en los libros tradicionales, disponibles en formato multimedia.

Tabla 4. Material 2. Análisis de material de un portal institucional

\begin{tabular}{ll}
\hline Dimensión & \multicolumn{1}{c}{ Principales características } \\
\hline Identificación & $\begin{array}{l}\text { Material Didáctico Digital y Objeto de Aprendizaje (Comunidad Valenciana). } \\
\text { Autoría: Carmen Coret Bueno. }\end{array}$ \\
\hline Estructura & Fácil uso, estructura encilla y tamaño adecuado. \\
\hline Tecnología & Imagen y sonido descargables. Velocidad de carga buena, rápida y sin problemas. \\
\hline Diseño & $\begin{array}{l}\text { Facilita la comprensión de contenidos. } \\
\text { Contenido organizado por familias de instrumentos y finaliza con la orquesta como un todo. }\end{array}$ \\
\hline Pedagogía & $\begin{array}{l}\text { Parte de conocimientos previos y plantea ampliación. } \\
\text { Contenidos relacionados con el entorno del alumnado y las características culturales de la } \\
\text { comunidad autónoma. } \\
\text { Modelo pedagógico activo, participativo, lúdico y motivador. }\end{array}$ \\
\hline Contenido & $\begin{array}{l}\text { Vídeo de concierto de orquesta, imágenes fijas de las cuatro familias de instrumentos y posición } \\
\text { de cada instrumento en la orquesta. La melodía utilizada pertenece a la "Guía d'orquestra per a } \\
\text { joves" de B. Britten. }\end{array}$ \\
\hline $\begin{array}{l}\text { Evaluación y } \\
\text { seguimiento }\end{array}$ & $\begin{array}{l}\text { Detalla criterios y estrategias de evaluación. } \\
\text { Actividades sencillas impresas: reconocimiento auditivo o asociación. No existe la opción de } \\
\text { crear recursos personalizables o de gestión de información. }\end{array}$ \\
\hline Fung
\end{tabular}

Fuente: Elaboración propia

El análisis del material de la Comunidad Valenciana revela que es una unidad didáctica breve con un recurso audiovisual que permite alcanzar los objetivos planteados; además es sencillo de utilizar, leer y aplicar. Propone diversas actividades, la mayoría grupales, por lo que adquiere peso el aprendizaje cooperativo, más allá del uso tecnológico. Como debilidades destacan: la no introducción de temas transversales; las músicas no son modelos próximos a la edad de los destinatarios del material; y la baja calidad de la imagen. Es fácilmente adaptable a contextos y niveles educativos similares, la metodología es cooperativa y activa facilitando la motivación e implicación del alumnado en el taller. Es un material válido en educación formal e informal. En cuanto a la presentación del recurso en la red educativa, es confusa ya que diferencia en la ventana principal tres actividades cuando en realidad dos de ellas se incluyen en la tercera. El taller se presenta bien organizado y tiene en cuenta los requisitos curriculares y organizativos, aunque no aprovecha toda su potencialidad para abordar contenidos transversales u otras áreas de conocimiento, no aborda usos o costumbres del contexto de la danza trabajada. Respecto a la propuesta de recursos y material didáctico, podría ser más atractiva y cercana a la

ROSA MARIA VICENTE ÁlVAREZ, DIANA MARÍN SUELVES Y OLGA CEPEDA ROMERO. THE CONTENT OF THIS ARTICLE IS THE SOLE RESPONSIBILITY OF THE AUTHORS. THE REVISTA ELECTRÓNICA DE LEEME AND UNIVERSITAT DE VALĖNCIA ARE NOT LIABLE FOR ANY LEGAL ACTIONS THAT MAY ARISE INVOLVING THE ARTICLE'S CONTENT. REVISTA ELECTRÓNICA DE LEEME - LISTA EIECTRONICA EUROPEA DE MUSICA EN LA EDUCACION- HTTP'//OIS UVES/INDEX. PHP/LEEME/INDEX ISSN: 1575-9563 EDITORES: UNIVERSIDAD DE VALENCIA Y JESÚS TEJADA GIMÉNEZ. VISIBILIDAD DE ESTA REVISTA: SCOPUS, EMERGING SOURCES CITATION INDEX (CLARIVATE), EBSCO, CINDOC (CSIC), CITEFACTOR, COPAC, DIALNET, DICE (CSIC), DOAJ, E-REVISTAS (CSIC), EBSCO PREMIER, ERIH+, GALE CENGAGE LEARNING, IN-RECS, IRESIE, LATINDEX, MIAR, OCLC WORLDCAT, RESH, REDIB, RILM CORE JOURNALS, SUDOC, ULRICHS, ESTA REVISTA ESTÁ PUBLICADA CON EL APOYO INSTITUCIONAL DE REDIRIS-CONSEJO SUPERIOR DE INVESTIGACIONES CIENTIFICAS Y ES DE ACCESO LIBRE. CREATIVE COMMONS LICENSE 4.O BY 
edad a la que va dirigida y el material no presenta personas con características similares a los destinatarios.

Tabla 5. Material 3. Análisis de material de una red de acceso abierto

\begin{tabular}{|c|c|}
\hline Dimensión & Principales características \\
\hline Identificación & Educación Artística. Música. Taller de Música. Acceso gratuito. \\
\hline Estructura & $\begin{array}{l}\text { Secuencia didáctica con tres actividades, planificada de una a tres horas. } \\
\text { Cada actividad plantea una breve descripción y acceso al recurso. }\end{array}$ \\
\hline Tecnología & $\begin{array}{l}\text { Buena navegabilidad, acceso y formatos, con iconos que acompañan al texto de función. } \\
\text { Características tecnológicas iguales a la plataforma de la red educativa excepto la actividad } 3 \text { en } \\
\text { la que se accede al portal de una organización determinada que presenta sus propias } \\
\text { características. }\end{array}$ \\
\hline Diseño & $\begin{array}{l}\text { Presentación iconográfica e hipertextual, intuitiva y muy accesible, únicamente por la vía visual } \\
\text { (no los recursos). }\end{array}$ \\
\hline Pedagogía & $\begin{array}{l}\text { Secuencia de actividades sin justificación ni argumentación. La actividad } 3 \text { supone un ejemplo } \\
\text { de taller donde se estructuran los elementos curriculares. } \\
\text { Presentación adecuada, síntesis del taller y puntos de ayuda para la comprensión de la actividad } \\
\text { (PDF). Propone criterios para incorporar la secuencia didáctica a la programación didáctica. } \\
\text { Contempla necesidades previas respecto a la competencia social y cívica y se relaciona con } \\
\text { cursos anteriores. Organiza el contenido de manera progresiva para el desarrollo de las } \\
\text { competencias. Propone una metodología activa y cooperativa, siendo el alumnado el motor y } \\
\text { actor principal de las actividades. } \\
\text { Explicita ámbito disciplinar y socio-afectivo, competencias básicas, objetivos de aprendizaje y } \\
\text { criterios de evaluación. Especifica organización de espacios y centra la atención en } \\
\text { determinadas situaciones sociales inadecuada para contrarrestarlas. El taller se desarrolla por el } \\
\text { grupo-clase en pequeños grupos que interactúan. }\end{array}$ \\
\hline Contenido & $\begin{array}{l}\text { Adaptada al nivel cognitivo del alumnado y la diversidad socio-cultural y política. El taller } \\
\text { plantea distintos niveles de exigencia. Presenta partitura de referencia a diferentes contextos. Es } \\
\text { adecuada para trabajar aspectos culturales. La propuesta sugiere agrupamiento en parejas por } \\
\text { género como criterio de agrupamiento. }\end{array}$ \\
\hline $\begin{array}{l}\text { Evaluación y } \\
\text { seguimiento }\end{array}$ & $\begin{array}{l}\text { Detalla criterios de evaluación por competencias. Presenta competencias, objetivos, contenidos } \\
\text { y evaluación del currículum. No especifica actividades de evaluación concretas. El desarrollo } \\
\text { de la actividad es un indicador de la consecución de los objetivos propuestos. }\end{array}$ \\
\hline
\end{tabular}

Fuente: Elaboración propia

\section{Discusión}

La práctica docente y los materiales didácticos digitales disponibles revelan que la tecnología ocupa un espacio principal en los espacios educativos (Peirats, Gallardo, San Martín y Waliño, 2016; Rodríguez y Rodríguez, 2015). Los materiales ejemplificados permiten obtener un análisis conjunto por sus características comunes: estar disponibles en plataformas educativas, dirigirse al alumnado de los cursos de quinto o sexto de Primaria y ser del área de educación musical. Además, responden a tres tipologías diferentes: plataforma institucional abierta, recurso de plataforma privada y libro de texto en formato digital. Estos materiales 
fueron desarrollados con el fin de ser utilizados para el alumnado. Los tres tienen componentes creativos que parten del profesorado, ya sea total o parcialmente, y son de uso frecuente en las escuelas.

Los materiales analizados suponen un cambio de formato, del papel al soporte digital. Sin embargo, el modelo educativo responde a un modelo tradicional y expositivo de enseñanza. Por tanto, el cambio de soporte no implica necesariamente innovación educativa, porque el modelo de enseñanza docente alentado no conlleva cambio metodológico. Algunos recursos (1 y 2) están dirigidos al alumnado. Otros (recurso 3) plantean su utilización en la planificación docente y sus características le confieren cierta licencia innovadora. Sin embargo, desde el punto de vista tecnológico, no suponen diferenciación ya que, presentados en formato tradicional, la práctica educativa sería similar.

Las editoriales desarrollan materiales idénticos al formato tradicional, aun cuando son materiales exclusivos para los medios tecnológicos. Los materiales de las webs institucionales (elaborados por docentes) refuerzan pedagogías y técnicas tradicionales. Estos materiales no aprovechan los recursos tecnológicos, están basados en la exposición de contenidos, y proporcionan información sobre la metodología conocida y utilizada por el profesorado de recursos digitales.

Dichos recursos basan sus tareas en el apoyo a la exposición magistral con presentaciones multimedia (Sutherland, Armstrong, Barnes, Brawn, Breeze, y Gall, 2004) y en el desarrollo de actividades individuales interactivas de bajo nivel de complejidad, como micro actividades a modo de los ejercicios de un libro de texto tradicional acompañadas de feedback inmediato, recursos multimedia como movimiento, sonido o animación, distribuidas en $C D$ $R O M$ o sitios web: ejercicios de puzles, test, asociaciones, sopas de letras, seriaciones, etc. La utilización del ordenador es puntual, más próximo a lo lúdico que a lo académico; además, son materiales complementarios o de ampliación de contenidos del libro de texto impreso. Encontramos tareas de interés formativo vinculadas a la competencia digital, pero se plantean de forma aislada y descontextualizada.

Aunque, asistimos a importantes políticas de inversión económica en infraestructuras, recursos tecnológicos y planes de formación del profesorado para el desarrollo de la competencia digital, así como en las webs institucionales (webs dinámicas, repositorios de materiales, etc.), los resultados revelan que el profesorado utiliza tecnología para apoyar pedagogías existentes (Balanskat, Blamire y Kefala, 2006; Fernández, Fernández y Rodríguez, 2017; López, 2017).

El análisis tecnológico revela formatos (PDF, Flash o HTML y HTML5) que no aprovechan su potencial técnico y basan su desarrollo en la exposición del contenido, tal y como se hace en los libros de texto tradicionales. La interacción en los ejercicios es limitada y de carácter individual. Los elementos multimedia son escasos y poco significativos. El desarrollo de estos materiales en formato digital se basa en aspectos lúdicos y llamativos. Los materiales son objetos digitales que complementan el texto y suelen tener un uso sencillo con baja calidad

Rosa MARÍA VICENTE ÁlVAREZ, Diana MARIN SUELVES Y Olga CEPEDA RoMero. THE CONTENT OF THIS ARTICLE IS THE SOLE RESPONSIBILITY OF THE AUTHORS. THE REVISTA ELECTRÓNICA DE LEME AND UNIVERSITAT DE VALËNCIA ARE NOT LIABLE FOR ANY LEGAL ACTIONS THAT MAY ARISE INVOLVING THE ARTICLE'S CONTENT REVISTA ELECTRÓNICA DE LEEME - USTA ELECTRÓNICA EUROPEA DE MÚSICA EN LA EDUCACIÓN- HTTP:/ /OJS.UVES/INDEXPHP/LFEME/INDEX ISSN: 1575-9563 EDITORES: UNIVERSIDAD DE VALENCIAY JESÚS TEJADA GIMÉNEZ. VISIBILIDAD DE ESTA REVISTA: SCOPUS, EMERGING SOURCES CITATION INDEX (CLARIVATE), EBSCO, CINDOC (CSIC), CITEFACTOR, COPAC, DIALNET, DICE (CSIC), DOAJ, E-REVISTAS (CSIC), EBSCO PREMIER, ERIH+, GALE CENGAGE LEARNING, IN-RECS, IRESIE, LATINDEX, MIAR, OCLC WORLDCAT, RESH, REDIB, RILM CORE JOURNALS, SUDOC, ULRICHS, ESTA REVISTA ESTÁ PUBLICADA CON EL APOYO INSTITUCIONAL DE REDIRIS-CONSEJO SUPERIOR DE INVESTIGACIONES CIENTIFICAS Y ES DE ACCESO LIBRE. CREATIVE COMMONS LICENSE $4.0 \mathrm{BY}$ 
de imagen baja. Los espacios de interacción social virtual son inexistentes o infrautilizados. Se ha impuesto el formato tradicional a pesar de desarrollar la didáctica a través de tecnología digital.

Los objetos digitales se encuentran en repositorios, clasificados en función de su potencial y contenido. El tipo de tareas ofrecidas dependen del potencial tecnológico. La finalidad preparatoria de otros materiales tecnológicos podría incorporar otra ventaja de la tecnología a través de vídeos explicativos o diagramas para el desarrollo de tareas físicas, así como otros recursos utilizables por el alumnado. En este sentido, los recursos tecnológicos deben posibilitar la organización de tareas que impliquen el uso de la tecnología a través de procesos de aprendizaje colaborativo entre el alumnado y entre clases virtuales.

\section{Conclusiones}

La utilización de tecnología en las aulas es una realidad que permite responder a diferentes demandas de la sociedad y del alumnado. Sin embargo, la existencia de diversidad de materiales didácticos digitales accesibles desde fuentes tecnológicas institucionales y editoriales no implica un cambio metodológico. El artículo presenta un análisis que revela MDD musicales con un método docente casi unitario, basado en la clase expositiva y en el desarrollo del trabajo individual, limitado a reproducir el recurso sin cuestionarlo. Frente a esto, los materiales y recursos didácticos digitales desarrollados y utilizados en el área de educación musical deben diseñarse desde una perspectiva que asiente prácticas docentes en el cambio metodológico y basada en pedagogías activas. Dichos materiales deben plantear tareas funcionales y significativas al alumnado. Además, el papel docente debe ser de guía, organización y supervisión de actividades de aprendizaje. La formación inicial y continua juega un papel clave en el proceso de selección y creación de materiales digitales adaptados al contexto y grupo clase.

La diversidad de prácticas con tecnología en educación musical revela el desarrollo de un modelo de integración didáctica poco intensa (Area, Hernández y Sosa, 2016). La figura docente con competencias digitales no debe desarrollarse únicamente en el cambio de soporte, debe ofrecer oportunidad de cambio educativo y desarrollar materiales con metodologías de enseñanza que cuestionen el monopolio del libro de texto como fuente única de conocimiento y estimular al profesorado en la búsqueda de nuevas estrategias, así como la reflexión y el contraste crítico permanente de los materiales.

El estudio presenta algunas limitaciones en cuanto a la muestra. Las tres comunidades autónomas del estudio constituyen el $18 \%$ del total del estado español y representan las diferencias existentes en España respecto a la implantación escolar de la tecnología educativa desde 2010. La selección de los materiales ha sido intencionada, respecto al interés de cada material dentro del estudio en razón de uso y difusión.

Rosa MARIA VICENTE ÁlVAREZ, DIANA MARÍN SUELVES Y OLGA CEPEDA ROMERO. THE CONTENT OF THIS ARTICLE IS THE SOLE RESPONSIBILITY OF THE AUTHORS. THE REVISTA ELECTRÓNICA DE LEEME AND UNIVERSITAT DE VALĖNCIA ARE NOT LIABLE FOR ANY LEGAL ACTIONS THAT MAY ARISE INVOLVING THE ARTICLE'S CONTENT. REVISTA ELECTRÓNICA DE LEEME - LISTA ELECTRÓNICA EUROPEA DE MÚSICA EN LA EDUCACIÓN-. HTTP://OJS.UV.ES/INDEX.PHP/LEEME/INDEX ISSN: 1575-9563 EDITORES: UNIVERSIDAD DE VALENCIA Y JESÚS TEJADA GIMÉNEZ. VISIBILIDAD DE ESTA REVISTA: SCOPUS, EMERGING SOURCES CITATION INDEX (CLARIVATE), EBSCO, CINDOC (CSIC), CITEFACTOR, COPAC, DIALNET, DICE SUDOC, ULRICHS, ESTA REVISTA ESTA PUBLICADA CON EL APOYO INSTITUCIONAL DE REDIRIS-CONSEJO SUPERIOR DE INVESTIGACIONES CIENTIFICAS Y ES DE ACCESO LIBRE. CREATIVE COMMONS LICENSE $4.0 \mathrm{BY}$ 
El instrumento de análisis, desarrollado por el equipo interdisciplinar, supone una herramienta de interés para el ámbito educativo (profesorado, editoriales e instituciones). Esta herramienta permite valorar materiales didácticos digitales en función de sus particularidades y necesidades. Pero es preciso analizar posibilidades y limitaciones de los recursos para tomar esta información como referencia en la contribución y desarrollo de materiales didácticos digitales innovadores.

Las líneas de investigación futura deberán profundizar en cambios epistemológicos como: conocer las diferencias entre los recursos ofertados en las diferentes comunidades autónomas, conocer el material didáctico digital disponible en las plataformas institucionales y el de las plataformas comerciales, así como su uso, preguntándonos: quién, cuándo, cómo o para qué (Vicente, 2017). Estas preguntas suscitan el desarrollo de líneas metodológicas como el estudio del uso del material didáctico digital en el proceso de enseñanza y aprendizaje, la formación en recursos digitales disponibles o los cambios que se producen en las prácticas educativas al introducir tecnología. Además, cabe la formulación de preguntas sobre la relación entre el éxito académico y el uso de los materiales didácticos digitales.

\section{Agradecimientos}

Al equipo de investigadoras e investigadores que formamos parte del Proyecto Escuel@ Digital, la escuela de la sociedad digital: análisis y propuestas para la producción y uso de los contenidos digitales educativos (EDU201564593-R), a los organismos públicos y a las editoriales que han permitido el análisis de sus materiales. Financiado por el Ministerio de Ciencia, Innovación y Universidades de España.

\section{Referencias}

Area, M. (2016). Guía de evaluación para el Estudio I. Análisis de la oferta de los recursos educativos en línea para el profesorado de educación Primaria. Guía evaluación Escuel@ Digit@ del Proyecto Escuel@ Digital (EDU2015-64593-R) Versión FINAL, financiado por el Plan Estatal de I+D+i. I. P. Manuel Area (Difusión restringida).

Area, M., Alonso, C., Correa, J. M., Del Moral, M. E., De Pablos, J., Paredes, J., Peirats, J., Sanabria, A. L., y San Martín, A. (2014). Las políticas educativas TIC en España después del Programa Escuela 2.0: las tendencias que emergen. RELATEC Revista Latinoamericana de Tecnología Educativa, 13 (2), 11-33. doi: 10.17398/1695-288X.13.2.11

Area, M., Hernández, V., y Sosa, J. J. (2016). Modelos de integración didáctica de las TIC en el aula. Comunicar: Revista Científica de Comunicación y Educación, 24 (47), 79-87. doi: 10.3916/C47-2016-08

Balanskat, A., Blamire, R. y Kefala, S. (2006). The ICT Impact Report. A review of studies of ICT impact on schools in Europe. European Schoolnet, European Comission. Recuperado de http://unpan1.un.org/intradoc/groups/public/documents/unpan/unpan037334.pdf

ROSA MARIA VICENTE ÁlVAREZ, DIANA MARIN SUELVES Y Olga CEPEDA ROMERO. THE CONTENT OF THIS ARTICLE IS THE SOLE RESPONSIBILITY OF THE AUTHORS. THE REVISTA ELECTRÓNICA DE LEEME AND UNIVERSITAT DE VALËNCIA ARE NOT LIABLE FOR ANY LEGAL ACTIONS THAT MAY ARISE INVOLVING THE ARTICLE'S CONTENT REVISTA ELECTRÓNICA DE LEEME - LISTA ELECTRÓNICA EUROPEA DE MUUSICA EN LA EDUCACIÓN- HTTP'//OJS.UVEES/INDEX.PHP/LEFME/INDEXISSN: 1575-9563 EDITORES: UNIVERSIDAD DE VALENCIAY JESÚS TEJADA GIMÉNEZ. VISIBILIDAD DE ESTA REVISTA: SCOPUS, EMERGING SOURCES CITATION INDEX (CLARIVATE), EBSCO, CINDOC (CSIC), CITEFACTOR, COPAC, DIALNET, DICE (CSIC), DOAJ, E-REVISTAS (CSIC), EBSCO PREMIER, ERIH+, GALE CENGAGE LEARNING, IN-RECS, IRESIE, LATINDEX, MIAR, OCLC WORLDCAT, RESH, REDIB, RILM CORE JOURNALS, SUDOC, ULRICHS, ESTA REVISTA ESTÁ PUBLICADA CON EL APOYO INSTITUCIONAL DE REDIRIS-CONSEJO SUPERIOR DE INVESTIGACIONES CIENTIFICAS Y ES DE ACCESO LIBRE. CREATIVE COMMONS LICENSE $4.0 \mathrm{BY}$ 
Bardin, L. (2004). Análise de conteúdo. Lisboa: Edições 70.

Cepeda, O., Gallardo, M ${ }^{\mathrm{a}}$ I. y Jesús Rodríguez (2017). La evaluación de los materiales didácticos digitales. RELATEC Revista Latinoamericana de Tecnología Educativa, 16 (2), 79 95. doi: 10.17398/1695-288X.16.2.79

Díaz, M. (1998). Materiales para la enseñanza de la música en la educación general. Revista de Psicodidáctica, 5, 83-94. Recuperado de http://www.redalyc.org/pdf/175/17517803009.pdf

Fernández, J. P. (2016). La adquisición y desarrollo de la competencia digital en alumnado de educación secundaria. Estudio de Caso. Cuadernos de Investigación Educativa, 7 (2), 83-98. doi: 10.18861/cied.2016.7.2.2612

Fernández, F. J., Fernández, M. J. y Rodríguez, J. M. (2018). El proceso de integración y uso pedagógico de las TIC en los centros educativos madrileños. Educación XX1, 21 (2), 395-416. doi: 10.5944/educXX1.17907

Fernández, A., Domínguez, E., y Armas, I. de. (2011). Herramienta para la revisión de la Calidad de Objetos de Aprendizaje Universitarios (COdA): guía del usuario. v.11 (Inédito). Recuperado de http://eprints.ucm.es/12533/

Giráldez, A. (2010). La composición musical como construcción: Herramientas para la creación y la difusión musical en Internet. Revista Iberoamericana de Educación, 52, 109-125. Recuperado de https://dialnet.unirioja.es/servlet/articulo?codigo=3157569

Jubany, J. (2010). Didáctica de la música en la educación obligatoria: recursos digitales y el caso del karaoke. Revista Eufonía, 50, 88-99.

López, N. (2017). Necesidades del profesorado especialista de Música de los centros de Primaria de Castilla La Mancha. Revista Electrónica de LEEME, 39, 74-102. doi: 10.7203./LEEME.39.9904

Oriol, N., Sustaeta, I. y Ráez, J. L. (1993). Guía de recursos didácticos. Madrid: Santillana.

Peirats, J. y Esnaola, G. A. (2015). Digitalización de los contenidos curriculares. Educatio Siglo XXI, 33 (3), 11-14. Recuperado de https://dialnet.unirioja.es/ejemplar/412130

Peirats, J., Gallardo, I. M., San Martín, A. y Waliño, M. J. (2016). Análisis de la industria editorial y protocolo para la selección del libro de texto en formato digital. Profesorado. Revista de Currículum y Formación del Profesorado, 20 (1), 75-89. Recuperado de https://recyt.fecyt.es/index.php/profesorado/article/view/49869/pdf 4

Pereiro, C. y Eirín, R. (2017). La estrategia institucional de la comunidad autónoma gallega para introducir las TIC en el ámbito educativo. Comunicación y Pedagogía, 299-300, 11-16. Recuperado de http://www.centrocp.com/

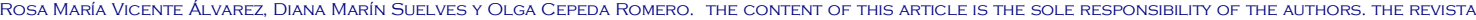
ELECTRÓNICA DE LEEME AND UNIVERSITAT DE VALĖNCIA ARE NOT LIABLE FOR ANY LEGAL ACTIONS THAT MAY ARISE INVOLVING THE ARTICLE'S CONTENT. REVISTA ELECTRÓNICA DE LEEME - LISTA ELECTRÓNICA EUROPEA DE MUSICA EN LA EDUCACIÓN- HTTP://OJS.UVES/INDEX PHP/LFEME/INDEX ISSN: 1575-9563 EDITORES: UNIVERSIDAD DE VALENCIA Y JESÚS TEJADA GIMÉNEZ. VISIBILIDAD DE ESTA REVISTA: SCOPUS, EMERGING SOURCES CITATION INDEX (CLARIVATE), EBSCO, CINDOC (CSIC), CITEFACTOR, COPAC, DIALNET, DICE (CSIC), DOAJ, E-REVISTAS (CSIC), EBSCO PREMIER, ERIH+, GALE CENGAGE LEARNING, IN-RECS, IRESIE, LATINDEX, MIAR, OCLC WORLDCAT, RESH, REDIB, RILM CORE JOURNALS, SUDOC, ULRICHS, ESTA REVISTA ESTÁ PUBLICADA CON EL APOYO INSTITUCIONAL DE REDIRIS-CONSEJO SUPERIOR DE INVESTIGACIONES CIENTIFICAS Y ES DE ACCESO LIBRE. CREATIVE COMMONS LICENSE 4.O BY 
Rodríguez, J. y Vicente, R. M. (2015). The music materials in early childhood education: a descriptive study in Galicia (Spain). International Journal of Music Education, 35 (2), 139-153. doi: $10.1177 / 0255761415619423$

Sanabria, A. L., Area, M. y Cepeda, O. (2012). El Proyecto Clic Escuela 2.0: El punto de vista del profesorado. En Actas del Congreso Internacional EDUTEC 2012: Canarias en tres continentes digitales: Educación, TIC, Net-Coaching (pp. 604-614). Las Palmas de Gran Canaria: Universidad de Las Palmas de Gran Canaria.

Sanabria, A.L. y Santana, P. (2017) (Coord.). Informe estudio I- Canarias del proyecto Escuel@ Digit@al (EDU2015-64593-R). La escuela de la sociedad digital: análisis y propuestas para la producción y uso de los contenidos digitales educativos. Madrid: Ministerio de Economía y Competitividad.

Santana, P. J., Eirín, R. y Marín, D. (2017). Análisis y evaluación de portales institucionales en España. Los casos de Canarias, Galicia y Valencia. RELATEC, Revista Latinoamericana de Tecnología Educativa, 16 (2), 29-48. doi: 10.17398/1695-288X.16.2.29

Strauss, A. y Corbin, J. (1998). Basics of qualitative research: Techniques and procedures for developing grounded theory. Thousand Oaks, CA: Sage.

Sutherland, R., Armstrong, V., Barnes, S., Brawn, R., Breeze, N. y Gall, M. (2004). Transforming teaching and learning: embedding ICT into everyday classroom practices. Journal of Computer Assisted Learning, 20 (6), 413-425. doi: 10.1111/j.1365-2729.2004.00104.x

Tejada, J. y Pérez, M. (2016). Diseño y evaluación de un programa informático para la educación musical de maestros no especialistas. El caso de EMOLab Revista Electrónica Complutense de Investigación en Educación Musical, 13, 1-28. doi: 10.5209/RECIEM.52072

Romero, J. (2003). Los medios y recursos para la educación musical en primaria. Tesis doctoral. Huelva: Universidad de Huelva.

Vicente, G. (2009). Movimiento y danza en Educación Musical: un análisis de los libros de texto de Educación Primaria (Tesis doctoral). Murcia: Universidad de Murcia. Recuperado de https://www.tesisenred.net/handle/10803/11076

Vicente, R. M. (2015). Educación infantil e música: os materiais dixitais na escola. Quinesia, 48-49, 88-110. Recuperado de https://issuu.com/revistadixitalquinesia/docs/

Vicente, R. M. (2017). Is there music in didactic materials? Searching for optimal learning experiences: an evaluation of music materials. En VVAA (2017). Early childhood music education in the Mediterranean. Raising children's musicality, evaluation music learning and enabling teachers preparation (pp. 207-220). Nicosia: Cyprus Centre for the Research and Study of Music. Recuperado de https://www.researchgate.net/publication/322056909

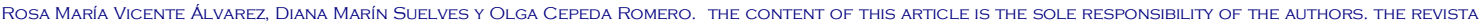
ELECTRÓNICA DE LEEME AND UNIVERSITAT DE VALĖNCIA ARE NOT LIABLE FOR ANY LEGAL ACTIONS THAT MAY ARISE INVOLVING THE ARTICLE'S CONTENT. REVISTA ELECTRÓNICA DE LEEME - LISTA ELECTRÓNICA EUROPEA DE MÚSICA EN LA EDUCACIÓN- HTTP'//OJS.UV ES/INDEX.PHP/LFEME/INDEX ISSN: 1575-9563 EDITORES: UNIVERSIDAD DE VALENCIA Y JESÚS TEJADA GIMÉNEZ. VISIBILIDAD DE ESTA REVISTA: SCOPUS, EMERGING SOURCES CITATION INDEX (CLARIVATE), EBSCO, CINDOC (CSIC), CITEFACTOR, COPAC, DIALNET, DICE (CSIC), DOAJ, E-REVISTAS (CSIC), EBSCO PREMIER, ERIH+, GALE CENGAGE LEARNING, IN-RECS, IRESIE, LATINDEX, MIAR, OCLC WORLDCAT, RESH, REDIB, RILM CORE JOURNALS, SUDOC, ULRICHS, ESTA REVISTA ESTÁ PUBLICADA CON EL APOYO INSTITUCIONAL DE REDIRIS-CONSEJO SUPERIOR DE INVESTIGACIONES CIENTIFICAS Y ES DE ACCESO LIBRE. CREATIVE COMMONS LICENSE $4.0 \mathrm{BY}$ 